\title{
Circulating spatial solitons
}

\author{
Eyal Feigenbaum and Meir Orenstein \\ Department of Electrical Engineering, Technion, Haifa 32000, Israel
}

Jacob Scheuer

Center for the Physics of Information, California Institute of Technology, Pasadena, California 91125

\begin{abstract}
Received November 3, 2005; accepted November 9, 2005; posted November 22, 2005 (Doc. ID 65495)
A class of optical spatial solitons exhibiting propagation in a closed-loop orbit in a two-dimensional plane is presented. A closed-form particlelike model is derived, indicating that the quasi-centrifugal force acting on these solitons can be balanced by an inhomogeneity in the nonlinear index of refraction. Specifically, a circular-shaped nonlinear interface is shown to facilitate stable orbital propagation of solitons that carve their own circular cavity for a wide range of nonlinearity parameters. (C) 2006 Optical Society of America OCIS codes: $190.5530,190.3270$.
\end{abstract}

One-dimensional (1D) spatial optical solitons (i.e., nonlinear confinement in one dimension) in Kerr media were discussed and measured in many publications. ${ }^{1-3}$ The dynamics of such solitons is characterized by linear trajectories within a twodimensional (2D) spatial plane (usually implemented as a slab waveguide). Introduction of material inhomogeneity (e.g., nonlinear interfaces) yields an equivalent force that modifies the soliton's trajectory. ${ }^{4-6}$ Here, by applying a circular interface (i.e., a disk) in a $2 \mathrm{D}$ plane, the respective soliton trajectories are bent to accommodate with a Keplerian-like orbit about the circumference of the nonlinear interface. Such solitons, upon completing a closed-loop trajectory, carve not only their selfwaveguides but also their self-circular ring cavity, with the potential for realization with relatively weak light sources.

Complex soliton trajectories, e.g., dipolelike propeller $(2 \mathrm{D}+1)$ incoherent spatial solitons (which selftrap in both transverse dimensions) were reported previously. ${ }^{1}$ These solitons exhibited a helical trajectory in a nonlinear medium. However, self-generation of a closed-loop trajectory, namely, self-carving of an optical cavity by a soliton, is novel to the best of our knowledge.

In the out-of-plane coordinate, referred to as $z$, the simplified $1 \mathrm{D}$ soliton is invariant, $\partial_{z}=0$. In the more general case the linear confinement along the $z$ axis (e.g., the slab waveguide mode) introduces a nonvanishing wavenumber $k_{z}$. This value of $k_{z}$ modifies the effective linear index of refraction and may impede the soliton stability. ${ }^{3,7}$ In the current analysis we employ a vanishing $k_{z}$ value and focus on the steadystate dynamics leading to the soliton capture into orbit.

Starting with the wave equation in polar coordinates $\{r, \theta\}$, we employ the slowly varying envelope amplitude $A$ under the paraxial approximation. An important distinction from the conventional approach is that we seek an angularly propagating nonlinear wave packet with a radial envelope, and thus the paraxial approximation is performed about the angular coordinate:

$$
\begin{aligned}
& \frac{\partial^{2} A}{\partial r^{2}}+\frac{1}{r} \frac{\partial A}{\partial r}+\frac{2 j k_{\theta}}{r^{2}} \frac{\partial A}{\partial \theta}+\left(k_{0}^{2} n_{0}^{2}+2 k_{0}^{2} n_{0} n_{2}|A|^{2}-\frac{k_{\theta}^{2}}{r^{2}}\right) A \\
& \quad=0
\end{aligned}
$$

where $k_{0}$ and $k_{\theta}$ are the free space and the angular wavenumbers, respectively, and $n_{0}, n_{2}$ are the respective linear and Kerr indices of refraction. By use of the conformal transformation $\{U=R \ln (r / R), V=R \theta\}$, the wave equation is transformed into

$$
\begin{aligned}
\frac{\partial A}{\partial V}= & \frac{j R}{2 k_{\theta}} \frac{\partial^{2} A}{\partial U^{2}}+\frac{j k_{0}^{2} n_{0} n_{2} R}{k_{\theta}} \exp \left(2 \frac{U}{R}\right)|A|^{2} A \\
& +\frac{j}{2}\left[\frac{k_{0}^{2} n_{0}^{2} R}{k_{\theta}} \exp \left(2 \frac{U}{R}\right)-\frac{k_{\theta}}{R}\right] A,
\end{aligned}
$$

where $R$ is an arbitrary scaling factor. Equation (2) may have general nonlinear wave solutions, but we seek solutions similar to the classical solitons of the nonlinear Schrödinger equation (NLSE). ${ }^{9}$ A formal NLSE can be obtained from Eq. (2) by employing a radially inhomogeneous nonlinear medium satisfying

$$
n_{0} \equiv \frac{k_{\theta}}{k_{0}} \frac{1}{r}, \quad n_{2} \sim \frac{1}{r}
$$

For such index profiles, Eq. (2) exhibits an exact orbiting soliton solution with a radial envelope proportional to $\operatorname{sech}[\log (r / R)]$.

Although the index profile of Eq. (3) supports an exact soliton solution, it is not practically realizable. Therefore, we seek circulating soliton solutions in a simpler configuration comprising two homogeneous nonlinear media that yield a stepwise inhomogeneity and focus our analysis on the interface as a source of soliton perturbation. To understand the required interface characteristics, we first examine Eq. (2) in a homogeneous medium, as a perturbed NLSE for a wave packet with a lateral width ( $U$ coordinate) significantly smaller than its orbital radius. For convenience we choose $R$ as the orbit radius. Equation (2) in normalized coordinates becomes 


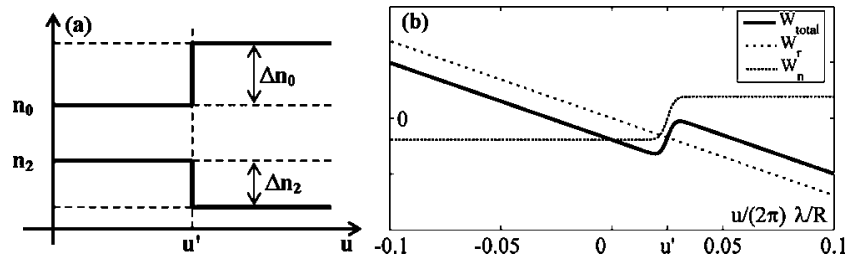

Fig. 1. (a) Refractive index profile $\left(n_{0}\right.$, linear; $n_{2}$, Kerr). (b) Potential well formation. $\epsilon=1,2 \pi R / \lambda_{0}=200, u_{0}=u^{\prime}=5, n_{0}$ $=1.45, n_{2}=0.1, \Delta n_{0}=0.1, \Delta n_{2}=0.05$.

$$
\frac{\partial}{\partial v} E=\frac{j}{2} \frac{\partial^{2} E}{\partial u^{2}}+j|E|^{2} E+j\left(n_{0}^{2}+2|E|^{2}\right) \frac{u}{k_{0} R} E,
$$

where

$$
\begin{gathered}
u \equiv k_{0} U, \quad v \equiv \frac{k_{0}^{2} R}{k_{\theta}} V, \\
A(u, v) \equiv \frac{E(u, v)}{\sqrt{n_{0} n_{2}}} \exp \left[-j\left(\frac{k_{\theta}^{2}}{2 k_{0}^{2} R^{2}}-\frac{n_{0}^{2}}{2}\right) v\right],
\end{gathered}
$$

Equation (4a) is a perturbed NLSE, which admits a perturbed soliton solution with intensity profile $|A|^{2}$ $=\epsilon^{2} \operatorname{sech}^{2}(\xi), \xi=\epsilon\left(u-u_{0}\right)$, where $\epsilon$ is the soliton peak amplitude and $u_{0}$ is the soliton center. By use of soliton perturbation theory, ${ }^{10}$ the perturbation source [the third term on the right-hand side of Eq. (4a)] introduces an effective force that accelerates the soliton outward:

$$
F^{r}=\frac{1}{k_{0} R}\left(n_{0}^{2}+\frac{2}{3} \epsilon^{2}\right) .
$$

The existence of this (fictitious) centrifugal force, $F^{r}$, in a homogeneous medium, is a manifestation of the fact that we imposed a circular motion on the soliton, which is following a linear trajectory in homogeneous media.

Thus, maneuvering the soliton into orbit requires an opposite force to compensate for $F^{r}$. Figure 1(a) describes the perturbation introduced by circular interface, with general variations in both linear and nonlinear refractive indices. When one applies the step index profile to the Kerr coefficient in Eq. (2), an additional perturbation source is formed in the modified perturbed nonlinear Schrödinger equation (4a):

$$
s^{n}=j \frac{\Delta\left(n_{0} n_{2}\right)}{n_{0} n_{2}} \mathbf{1}\left(u-u^{\prime}\right)|E|^{2} E, \quad \mathbf{1}\left(u-u^{\prime}\right) \equiv \begin{cases}1 & u>u^{\prime} \\ 0 & u<u^{\prime}\end{cases}
$$

where $\quad \Delta\left(n_{0} n_{2}\right) \equiv\left(n_{0}+\Delta n_{0}\right)\left(n_{2}-\Delta n_{2}\right)-n_{0} n_{2}$. The equivalent force acting on the soliton due to this disk interface is a function of the soliton displacement from this interface:

$$
F^{n}=\frac{\epsilon^{3} \Delta\left(n_{0} n_{2}\right)}{4\left(n_{0} n_{2}\right)} \operatorname{sech}^{4}\left[\epsilon\left(u^{\prime}-u_{0}\right)\right] .
$$

To set the soliton in a stable orbit, the total applied force must vanish at a certain radius, around which an equivalent mechanical potential well is formed. Figure 1(b) illustrates the potential well shape, $W=-\int F \mathrm{~d} u$, and its two constituents, $W^{r}$ and $W^{n}$, related to $F^{r}$ and $F^{n}$, respectively. Since the potential minimum is local, the capture of a soliton in orbit is possible if the initial position of a stationary soliton is within this local well. Solitons that are initially outside will be swept away from the interface and escape unless sufficient equivalent kinetic energy that is related to their initial radial velocity $\left(k_{u}\right)$ is provided to them. The attracting force in real space is scaled by $1 / k_{\theta}^{2}$, thus higher angular velocity (which is proportional to $k_{\theta}$ ) reduces the depth of the potential well.

The necessary conditions for obtaining closed-loop orbital motion are found by equating the two radial forces acting on the soliton and using the fact that $\operatorname{sech}^{4}$ is bounded by 0 and 1 :

$$
0 \leqslant \frac{n_{0}^{2}+\frac{2}{3} \epsilon^{2}}{k_{0} R} \frac{4\left(n_{0} n_{2}\right)}{\epsilon^{3} \Delta\left(n_{0} n_{2}\right)} \leqslant 1 .
$$

The left inequality corresponds to $\Delta\left(n_{0} n_{2}\right)$ being positive. A particularly interesting case is the one of zero (no interface for low intensity) or positive $\Delta n_{0}$ for which there is no linearly confined mode in the disk. For this case, the Kerr coefficient of the inner (disk) material should be higher than that of the cladding material. From the right inequality we extract a threshold value for $\Delta n_{2}$, which is a necessary condition for supporting an orbital soliton:

$$
\min \left(\Delta n_{2}\right)=\frac{n_{2}}{n_{0}} \Delta n_{0}+\frac{4 n_{2}\left(n_{0}^{2}+\frac{2}{3} \epsilon^{2}\right)}{k_{0} R \epsilon^{3}} .
$$

We compare our closed-form results with angular beam propagation simulations based on the NLSE. ${ }^{9}$ For a linear positive index step height $\left(\Delta n_{0}\right)$ and without nonlinear step $\Delta n_{2}$, the soliton escapes.
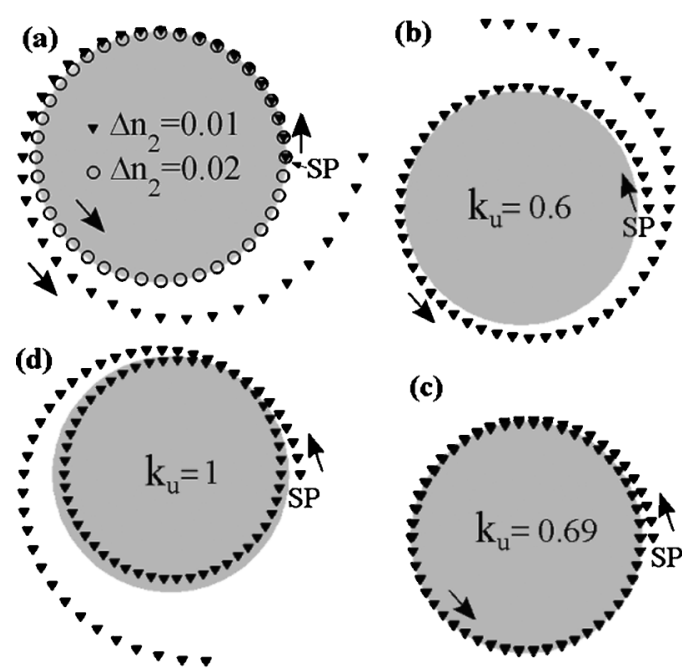

(c)

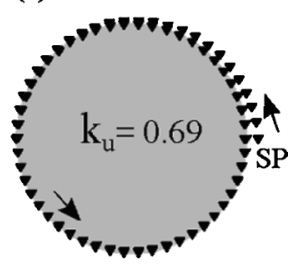

Fig. 2. Simulation trajectories. $\epsilon=1, R=2 \pi \times 100 \lambda_{0}, u^{\prime}$ $=0, n_{0}=1.45, n_{2}=0.1, k_{\theta}=1000$. (a) $k_{u}=0, u_{0}=0, \Delta n_{0}=0.05$. (b) - (d) $\Delta n_{0}=0.01, u_{0}=10, \Delta n_{2}=0.03$. SP, starting point. 

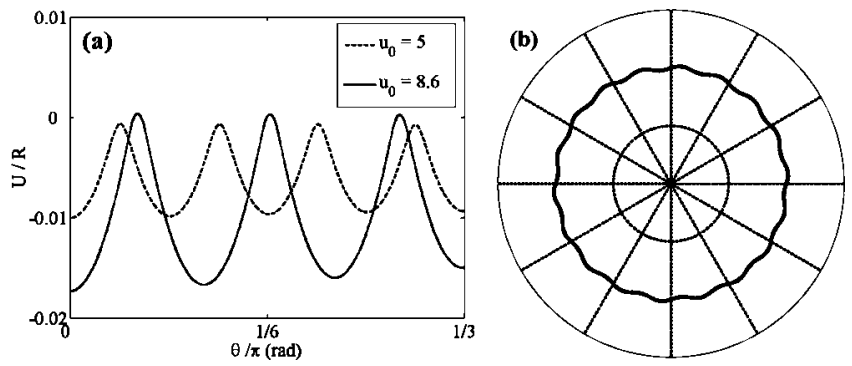

Fig. 3. (a) Closed-loop oscillating soliton trajectories for initial shifts of 5 (dashed curve) and 8.6 (solid curve). (b) Polar view of the latter. The structure parameters are $R k_{0}=500, n_{01}=1.5, n_{02}=1.45$, and $n_{21}=n_{22}=0.0625$.

When the nonlinear step exceeds a threshold level, the soliton is captured into a circular trajectory. Equation (9) predicts quite accurately the threshold value of the index step obtained by simulations, and the agreement improves for larger $k_{0} R$ values. The threshold value depends inversely on the radius because the centrifugal force $\left(F^{r}\right)$ decreases for a larger orbital radius, and a smaller balancing force $\left(F^{n}\right)$ is required. Increasing the negative linear index step value necessitates a corresponding increase of the nonlinear step to keep the soliton in orbit.

Figure 2(a) depicts the formation of the orbiting soliton in the vicinity of an index step. Soliton trajectories extracted from several beam propagation simulations are shown. They verify that orbital solitons can evolve even for an opposite (repelling) linear index of refraction [shown schematically in Fig. 1(a)]. Contrary to regular linear wave packets, which would definitely escape orbit for such a linear refractive index profile, the soliton self-carves a circular ring cavity as a result of nonlinear effects. Decreasing the Kerr index difference would eventually eliminate the potential well in the vicinity of the interface and cause the soliton to escape. In Figs. 2(b)-2(d) the soliton is initially displaced from the interface but is provided with initial "kinetic energy," namely, a radial velocity (wavenumber in the radial direction, $k_{u}$ ). Three trajectories are depicted for three different initial $k_{u}$ starting at the same initial spatial location. For $k_{u}=0.69$ the soliton is trapped in orbit.

The potential well analogy allows the identification of more-complex trajectories than cyclic ones. Any initial deviation of the soliton from the center of the well results in oscillations around the orbit. To fulfill the requirement of a closed-loop trajectory, the oscillations must complete an integer number of periods in a single round trip. These oscillations, similar to the light-ray trajectories of geometrical optics, were revealed in our simulation but exhibited a relatively small amplitude compared with orbital radius (see Fig. 3). Figure 3 depicts two examples for oscillating orbital solitons. Because the induced potential well is inharmonic, the oscillation period depends on the oscillation amplitude (i.e., the initial shift from the minimum of the potential well), and as a result only discrete values of the initial shift can satisfy the oscillation condition.

To transform the simulation results back to polar coordinates, $\{r, \theta\} k_{\theta}$ should be determined:

$$
k_{0} r=\left(k_{0} R\right) \exp \left(\frac{u}{k_{0} R}\right), \quad \theta=\frac{k_{\theta}}{\left(k_{0} R\right)^{2}} v .
$$

The effect of the self-cavity, manifested by the periodicity of the angular trajectory, is obscured by the coordinate transformation. Transforming the soliton solution back to the conventional cylindrical coordinates requires direct application of these cyclic boundary conditions, resulting in an integer-valued angular $k$-number $\left(k_{\theta}\right)$ and a requirement for an integer number of amplitude periods, $\theta_{\text {per }}$ (corresponding to $\left.v_{\text {per }}\right)$, in one cycle $(2 \pi)$. This leads to discrete values of $k_{0} R$ that basically determine the resonance wavelength of the nonlinear cavity:

$$
m k_{\theta}=l=\frac{2 \pi\left(k_{0} R\right)^{2}}{v_{\text {per }}},
$$

where $m$ and $l$ are integers and $v_{\text {per }}$ is obtained from the simulation for each $k_{0} R$.

To conclude, we have presented a new class of solitons circulating in a closed-loop orbit. The centrifugal force of the solitons is balanced by an attractive central force induced by a nonlinear circular interface. Such orbital solitons exist for a vanishing and even for a repelling linear step index profile, given that the nonlinear index difference is high enough. The threshold value of the onset of orbital motion was calculated and confirmed by numerical simulation. This threshold can be exploited to achieve a soliton intensity discriminator that deflects high-intensity beams into a locked circular motion while preserving the cross-field propagation of a lower-intensity beam. The stability of the soliton circular motion and additional interesting cavity related dynamics (possible bistability due to soliton energy storage) are subjects for further research.

M. Orenstein's e-mail address is meiro @ee.technion.ac.il.

\section{References}

1. G. I. Stegeman and M. Segev, Science 286, 1518 (1999).

2. Y. S. Kivshar and G. I. Stegeman, Opt. Photonics News 13, 59 (2002).

3. B. A. Malomed, D. Mihalache, F. Wise, and L. Torner, J. Opt. B: Quantum Semiclassical Opt. 7, R53 (2005).

4. J. Scheuer and M. Orenstein, Opt. Lett. 24, 1735 (1999).

5. E. Alvarado-Méndez, G. E. Torres-Cisneros, M. TorresCisneros, J. J. Sánchez-Mondragón, and V. Vysloukh, Opt. Quantum Electron. 30, 687 (1998).

6. A. B. Aceves, P. Varatharajah, A. C. Newell, E. M. Wright, G. I. Stegeman, D. R. Heatley, J. V. Moloney, and H. Adachihara, J. Opt. Soc. Am. B 7, 963 (1990).

7. L. Djaloshinski and M. Orenstein, IEEE J. Quantum Electron. 35, 737 (1999).

8. M. Heiblum and J. H. Harris, IEEE J. Quantum Electron. 11, 75 (1975).

9. G. P. Agrawal, Nonlinear Fiber Optics, 2nd ed. (Academic, New York, 1995).

10. H. A. Haus and W. S. Wong, Rev. Mod. Phys. 68, 423 (1996). 